CIRJE-F-1010

\title{
Style Analysis with Particle Filtering and Generalized Simulated Annealing
}

\author{
Takaya Fukui \\ Graduate School of Economics, The University of Tokyo \\ Seisho Sato \\ The University of Tokyo \\ Akihiko Takahashi \\ The University of Tokyo
}

April 2016; revised in June 2016 and March 2017

CIRJE Discussion Papers can be downloaded without charge from:

http://www.cirje.e.u-tokyo.ac.jp/research/03research02dp.html

Discussion Papers are a series of manuscripts in their draft form. They are not intended for circulation or distribution except as indicated by the author. For that reason Discussion Papers may not be reproduced or distributed without the written consent of the author. 


\title{
Style Analysis with Particle Filtering and Generalized Simulated Annealing *
}

\author{
Takaya Fukui, Seisho Sato, Akihiko Takahashi \\ Graduate School of Economics, The University of Tokyo
}

March 9, 2017

\begin{abstract}
This paper proposes a new approach to style analysis of mutual funds in a general state space framework with particle filtering and generalized simulated annealing (GSA). Specifically, we regard the exposure of each style index as a latent state variable in a state space model and employ a Monte Carlo filter as a particle filtering method, where GSA is effectively applied to estimating unknown parameters.

An empirical analysis using data of three Japanese equity mutual funds with six standard style indexes confirms the validity of our method. Moreover, we create fund-specific style indexes to further improves estimation in the analysis.
\end{abstract}

Keyword: Style analysis, Particle filtering, Monte Carlo filter, Generalized simulated annealing, Mutual fund, State space model

*This research is supported by CARF (Center for Advanced Research in Finance) and JSPS KAKENHI (Grant Number 25380389). 


\section{Introduction}

It has been widely recognized particularly since the seminal work of Sharpe (1992) that style analysis is an important process to select investment funds as well as to monitor and evaluate funds' actions, risks and performances.

For instance, it helps to clarify which fund's style conforms to an investor's own investment goal. Moreover, although each fund's investment report normally discloses its one-shot composition only annually or semiannually, ${ }^{1}$ the return-based analysis with properly chosen style factors enables us to assess funds' risk-return profiles on a daily basis in a unified and efficient manner. For example, one may decide and change allocations to multiple equity funds by applying a style analysis with common stock indexes, which represent investment categories such as large/middle/small cap stocks, growth/value stocks and their combinations.

Also, investors are able to check and adjust their style factors and the estimated exposures periodically by consulting funds investment reports. Conversely, one might examine whether reported one-shot compositions at specific timings are adequate, as compared to the past sequences of the one's own estimates.

In the current paper, we propose a new framework for style analysis based on a Monte Carlo filter with generalized simulated annealing (GSA). While a general state space model and a Monte Carlo filter are expected to be powerful tools for style analysis, they are rarely applied to estimations of mutual funds' styles. As an example of their financial applications, Takahashi and Sato (2001) successfully developed a method for empirical analysis of the term structure models. This paper develops a state space model for return-based style analysis of mutual funds with applying GSA to estimation of model parameters.

Firstly, we suppose that there are $n$ style indexes and that the rate of return (from $t$ to $t+\Delta t$ ), $r_{t}$ of a mutual fund is approximately expressed as

\footnotetext{
${ }^{1}$ Typically, these reports are published in a few months after each fund's settlement dates.
} 
a portfolio consisting of these indexes.

$$
r_{t}=\sum_{i=1}^{n} \beta_{i t} I_{i t}+u_{t}
$$

In the equation, $I_{i t}$ denotes a rate of return (from $t$ to $t+\Delta t$ ) of a style index $i$, the coefficient $\beta_{i t}$ represents the so called style weight at $t$ for the index $i$, and $u_{t}$ is a residual. Estimating the coefficients $\beta_{i t}, i=1, \cdots, n$ by using observational data is main objective for style analysis. If each $\beta_{i t}$ is supposed to be invariant over time that is $\beta_{i t}=\beta_{i}$, the problem is reduced to a regression analysis under constraints that all $\beta_{i}$ are non-negative and the sum of $\beta_{i} i=1, \cdots, n$ is equal to one; that is $\beta_{i} \geq 0$ for all $i=1, \cdots, n$ and $\sum_{i=1}^{n} \beta_{i}=1$. These constraints reflect the assumption that the fund is a portfolio of $n$ style indexes and only long positions are admitted. Then, the coefficients can be estimated by a least square method with constraints. This approach was initiated by Sharpe (1992). His framework is widely used in practice as well as in academic research; for instance, see Busse (1999), Fung and Hsieh (1997) and Chan, Chen and Lakonishok (2002). From a different viewpoint, Brown and Goetzmann (1997) and Pattarina, Paterlinib and Minervac (2004) proposed a new clustering method for style analysis, which did not estimate the style weights.

As argued by Grinblatt, Titman and Wermers (1995) and Ferson and Schadt (1996), it may not be acceptable to apply the models with timeinvariant weights to actual funds' data because a portfolio manager dynamically change the portfolio weights. Although Sharp (1992) tried to estimate the coefficients by using a window regression in order to capture dynamic variation of the weights of indexes, it is a hard task to determine the optimal width of the window. Also, theoretically speaking, it is not appropriate to estimate the coefficients $\beta_{i t}$ in the equation (1) by a window regression, because there are too few observations relative to the number of the coefficients $\left(\beta_{i t}\right)$. An estimation for this type of models is made possible by assuming a time series structure in time-varying coefficients based on a Bayesian framework. In particular, we regard the coefficients of styles as state variables and 
regard a fund's return and style indexes as observations. Thus, the current paper proposes a new approach based on a general state space model and a Monte Carlo filter frequently used in Bayesian frameworks.

In a Bayesian approach, although Swinkels and van der Sluis (2006) and Bodson, Coën, and Hübner (2010) presented applications of Kalman filter for the estimation of time-varying weights, they ignore non-negative constraints of weights. Marques, Pizzinga and Vereda (2012) used a restricted Kalman filter to satisfy the constraints. However, it is almost impossible to trace sudden changes in the weights by those existing methods, which was pointed out by Annaert and Campenhout (2007).

In order to overcome the problems, a previous unpublished discussion paper, Kobayashi, Sato and Takahashi (2005) proposed a style analysis by utilizing a state space model. As a subsequent work, the current paper presents a new framework for style analysis based on a general state space model: it develops a system model with the non-negative constraints of weights which allows compound Poisson system noises in addition to Gaussian noises, proposes a new estimation method that is, a Monte Carlo filter with generalized simulated annealing (GSA), and implements an empirical analysis using different typical Japanese mutual funds' recent data with our own fund-specific style indexes.

Specifically, for estimating parameters in the state space model, our GSA method is more efficient than a grid search and a self-organizing method used in the previous work, which will be explained to the detail in Section 3.2. Further, in the empirical analysis, through examining investment reports of the mutual funds in detail, we find that existing indexes are not appropriate for the style analysis of some mutual funds. Even for those cases, we are able to create more adequate ones based on the reports, and confirm that our method is very effective in practical style analysis by using the new indexes, which will be clarified in Section 4.2.

The paper is organized as follows. The next section overviews a general state space model related to this study. Section 3 explains a modeling frame- 
work for style analysis with several concrete examples useful in applications. Then, we introduce a generalized simulated annealing (GSA) method for estimation of parameters in our models. Section 4 tests the validity of our method by using historical data of three Japanese mutual funds with existing and our newly developed style indexes. Section 5 concludes. Appendix 1 provides a typical algorithm of Monte Carlo filtering, and Appendix 2 describes the method applied to our empirical analysis step by step.

\section{State Space Modeling}

In this section, we introduce a general state space model and a Monte Carlo filter, which will be applied to developing a new method for style analysis of mutual funds in the following sections.

Suppose that some observations are given and that they are determined by unobservable important variables called "state variables" as well as by observable exogenous variables and noises. In order to estimate state variables, a state space model consisting of a system model and an observation model is very effective. A system model determines the dynamics or time series structures of state variables where Markovian structure is assumed, while an observation model mainly describes the relation between observation variables and the state variables.

We introduce a state space model that consists of the following system model and the observation model:

$$
\left\{\begin{aligned}
X_{t} & =F\left(X_{t-\Delta t}, v_{t}\right) \quad(\text { system model }) \\
Z_{t} & =H\left(X_{t}\right)+u_{t} \quad \text { (observation model) }
\end{aligned}\right.
$$

where $X_{t}, Z_{t}$ and $\Delta t$ denote a $N$ dimensional state vector, a $M$ dimensional observation vector at time $t$ and the time interval of observational data, respectively while $v_{t}$ and $u_{t}$ denote the system noise and the observational noise whose density functions are given by $q(v)$ and $\psi(u)$ respectively. $F$ and $H$ are generally non-linear functions of $R^{N} \times R^{N} \mapsto R^{N}$ and $R^{N} \mapsto R^{M}$, and the initial state vector $X_{0}$ is assumed to be a random variable whose 
density function is given by $p_{0}(X)$.

Further, in order to handle the cases that explicit functional relations such as $F$ and $H$ cannot be obtained, we can introduce a more general state space model based on conditional distributions:

$$
\left\{\begin{aligned}
X_{t} & \sim \mathbf{F} \mid X_{t-\Delta t} \quad \text { (system model) } \\
Z_{t} & \sim \mathbf{H} \mid X_{t} \quad \text { (observation model) }
\end{aligned}\right.
$$

where $\mathbf{F} \mid X_{t-\Delta t}$ and $\mathbf{H} \mid X_{t}$ denote a conditional distribution of $X_{t}$ given $X_{t-\Delta t}$ and that of $Z_{t}$ given $X_{t}$ respectively. See Kitagawa and Gersh (1996) for more details of a state space model.

In style analysis we can observe only the returns of a fund, and our objective is to estimate time-varying style exposures by using style indexes. In the framework of state space model, the equation (1) is thought as an observation model. Then we regard style exposures and style indexes as unknown state variables and observable exogenous variables respectively in the equation. In modeling dynamics of style exposures in the system model, we need to consider the restriction of style exposures such that they should be nonnegative and the sum of them must be equal to one. Moreover, we take into account the possibility of sudden changes of style exposures. Our general state space modeling approach has an advantage in a sense that various types of system models can be applied. For example, we may utilize a compound Poisson or a normal mixture distribution of the system noise to express sudden changes of style exposures. However, the resulting system and observation models become nonlinear and non Gaussian, which requires special consideration in estimation.

Our task is to estimate unobservable state variables $X$ through observable variables $Z$. We need to notice that the standard Kalman filter cannot be justified in the situation that the system or the observation models are non-linear or non-Gaussian. Instead a particle filter is a powerful estimation technique that can be applied to the difficult situation.

While several approaches are proposed for particle filter (see Doucet, Barat, and Duvaut (1995), Durbin, and Koopman (1997), Gordon, Salmond, 
and Smith (1993), Tanizaki (1993), and Doucet, de Freitas and Gordon, eds. (2001) for instance.), we take a Monte Carlo filter developed by Kitagawa (1996), which emphasizes practical applications and presents the concrete computational procedures under fairly general assumptions. (A good example is Higuchi (1999).) Following the approach, we approximate the distribution of a state vector $X_{t}$ by many realizations drawn from that distribution. We call those realizations as "particles". Given $m$ particles of a state vector $\left\{\xi_{t-\Delta}^{[1]}, \cdots, \xi_{t-\Delta}^{[m]}\right\}$, we can obtain a one step ahead predictor from the system model (2) as a set of particles:

$$
p_{t}^{[k]}=F\left(\xi_{t-\Delta}^{[k]}, v_{t}^{[k]}\right) .
$$

Then we have a filter distribution of $X_{t}$ through resampling from the predictor distribution $\left\{p_{t}^{[1]}, \cdots, p_{t}^{[m]}\right\}$. In resampling, a higher weight is assigned on $p_{t}^{[k]}$ which fits better to a given observation $Z_{t}$. The resampled particles denoted by $\left\{\xi_{t}^{[1]}, \cdots, \xi_{t}^{[m]}\right\}$ are regard as the filter estimates of $X_{t}$. We repeat these steps up to $T$. Appendix 1 provides more explanations and an algorithm of Monte Carlo filtering. Moreover, Appendix 2 describes how to apply the general method to a practical example of style analysis.

\section{Style Analysis in a General State Space Model}

This section explains an application of state space modeling to style analysis for mutual funds and introduces generalized simulated annealing (GSA) for the parameters' estimation.

\subsection{Modeling Framework for Style Analysis}

First, we consider a system model. Essentially, we regard the coefficients of style indexes denoted by $\beta_{i t}, i=1, \cdots, n$ in the equation (1) as state variables which follow stochastic processes with constraints. Moreover, we do not model $\beta_{i t}$ directly, but introduce more fundamental state variables $Y$ behind $\beta_{i t}$ which determines the dynamics of $\beta_{i t}$. 
Let us define $\mathbf{R}^{n+k}$-valued state variables $X_{t}=\left(Y_{t}, \beta_{t}\right)^{\prime}$, where the $\mathbf{R}^{k}$ valued state variables $Y_{t}$ follow

$$
Y_{t}=f\left(Y_{t-\Delta t}, \beta_{t-\Delta t}, t\right)+v_{t}
$$

Here,

$$
\begin{aligned}
& Y_{t}=\left(Y_{1 t}, \cdots, Y_{k t}\right), \\
& \beta_{t}=\left(\beta_{1 t}, \cdots, \beta_{n t}\right),
\end{aligned}
$$

and the system noise $v_{t}$ follows a distribution $A$ whose density function is given by $q(v)$ :

$$
v_{t} \sim \text { distribution } A \text {. }
$$

This system model includes the model such that the current $Y_{t}$ depends not only on $Y_{t-\Delta t}$ in the previous period, but also on $\beta_{t-\Delta t}$. On the other hand, the state variables $\beta_{i t}, i=1, \cdots, n$ are determined based on the current $Y_{t}$ so that $\beta_{i t}$ satisfy the constraints:

$$
\begin{aligned}
& \beta_{i t} \geq 0 \text { for all } i=1, \cdots, n, \\
& \sum_{i=1}^{n} \beta_{i t}=1 .
\end{aligned}
$$

We are able to introduce various formulations for the relation between $\beta_{t}$ and $Y_{t}$. In particular, we show two examples below.

(i) The first example describes the case that an analytic expression between these variables can be obtained. For instance, $\beta_{i t}, i=1, \cdots, n$ are given by

$$
\beta_{i t}=h_{i}\left(Y_{t}, t\right), i=1, \cdots, n,
$$

where $h_{i}\left(Y_{t}, t\right), i=1, \cdots, n$ are $\mathbf{R}$-valued some functions of $Y_{t}$ and $t$, so that the constraints are satisfied. For instance, we can use a logit transformation. That is, $\beta_{i t}, i=1, \cdots, n$ are determined by

$$
\beta_{i t}=h_{i}\left(Y_{t}, t\right)=\frac{e^{Y_{i t}}}{\sum_{i=1}^{n} e^{Y_{i t}}} .
$$


Hence, in this case the system equation is expressed as follows:

$$
\begin{aligned}
& Y_{t}=f\left(Y_{t-\Delta t}, \beta_{t-\Delta t}, t\right)+v_{t}, \\
& \beta_{i t}=h_{i}\left(Y_{t}, t\right)=\frac{e^{Y_{i t}}}{\sum_{i=1}^{n} e^{Y_{i t}}}, i=1, \cdots, n,
\end{aligned}
$$

the system noise $v_{t} \sim$ distribution $A$.

We note that the equation (5) corresponds to $F(\cdot)$ in the equation $(2)$ of the state space model. The functional form in the equation (5) reflects our assumption stated before that the fund can be regarded as a portfolio of style indexes with long positions. Those constraints can be captured by the functions $h_{i}(\cdot), i=1, \cdots, n$ such as in (5). We also note that $\beta_{t}$ and $Y_{t}$ are estimated by using a Monte Carlo filter since they are not observable, the functions $f$ and $h$ are non-linear in general, and the system noise $v_{t}$ may follow a non-normal distribution.

(ii) In the second example, we apply models to the case that it is difficult to capture the required constraints as explicit functions. In the case, we go back to a general state space framework introduced in the equation (3). We first notice that the condition $\sum_{i=1}^{n} \beta_{i t}=1$ allows us to reduce the dimension of $\beta=\left(\beta_{1}, \cdots, \beta_{n}\right)$ from $n$ to $n-1$. Next we fix some $j \in\{1,2, \cdots, n\}$ and introduce the notation $x^{(j)}$ as a vector of which the $j$-th element is removed from a vector $x$. For example, $\beta_{t}^{(j)}$ is defined as

$$
\beta_{t}^{(j)} \equiv\left(\beta_{1 t}, \cdots, \beta_{j-1 t}, \beta_{j+1 t}, \cdots, \beta_{n t}\right)
$$

Let $k=n$, and hence $Y_{t}=\left(Y_{1 t}, \cdots, Y_{n t}\right)$. Next, let some $j \in\{1, \cdots, n\}$ fixed. For each $i=1, \cdots, n, i \neq j$, we assume that $Y_{i t}$ is generated by the equation:

$$
Y_{i t}=f_{i}\left(Y_{t-\Delta t}, \beta_{t-\Delta t}, t\right)+v_{i t} .
$$

Let us define a set $A_{t}^{(j)}$ as

$$
A_{t}^{(j)}=\left\{0 \leq Y_{1 t}, \cdots, 0 \leq Y_{j-1 t}, 0 \leq Y_{j+1 t}, \cdots, 0 \leq Y_{n t}, \sum_{i \neq j} Y_{i t} \leq 1\right\} .
$$


Then, $\beta_{t}^{(j)}$ is generated according to the distribution function $G\left(y^{(j)}\right)$ which is defined by

$$
G\left(y^{(j)}\right)=\operatorname{Pr}\left(\left\{Y_{t}^{(j)} \leq y^{(j)}\right\} \mid A_{t}^{(j)}\right)=\frac{\operatorname{Pr}\left(\left\{Y_{t}^{(j)} \leq y^{(j)}\right\} \cap A_{t}^{(j)}\right)}{\operatorname{Pr}\left(A_{t}^{(j)}\right)}, y^{(j)} \in \mathbf{R} .
$$

Finally, $\beta_{j t}$ is determined by

$$
\beta_{j t}=1-\sum_{i \neq j} \beta_{i t}
$$

Moreover, in order to avoid any bias caused by $j$ being fixed, we randomly choose $j \in\{1, \cdots, n\}$ with probability $\frac{1}{n}$ at each time point $t$.

In the following section, we will apply the example (i) as well as the example (i) combined with the example (ii) to an empirical analysis.

Finally, let us consider the observation equation. That is, a fund return $r_{t}$ is determined by

$$
r_{t}=\sum_{i=1}^{n} \beta_{i t} I_{i t}+u_{t}
$$

where $I_{i t}, i=1, \cdots, n$ represent style index returns and the observation noise $\left(u_{t}\right)_{t}$ follows a distribution $B$ of which density function is given by $\psi(u)$,

$$
\left(u_{t}\right)_{t} \sim \text { distribution } B
$$

The equation (9) corresponds to $H(\cdot)$ in the equations (2) of the state space model. Here, we note that the fund return $r_{t}$ and style index returns $I_{i t}$, $i=1, \cdots, n$ are obtained as observations.

\subsection{Estimation Method for Parameters}

This subsection explains an estimation method for parameters in models, which are variances of system noises in our model for an empirical study in the next section.

We apply the maximum likelihood estimation (MLE) method in parameter estimation. It is well known that because a likelihood function 
obtained by a Monte Carlo filter is not smooth in the parameters mainly due to the Monte Carlo noises, optimization methods based on gradients and numerical differentiations cannot be applied. While a grid search is an alternative method, it generally takes high computational costs to determine an adequate parameter space before the search. Although we may use self-organization method, where unknown parameters regarded as state variables are time variant, we have difficulty in interpretation of the obtained parameters.

To avoid problems above, we employ a generalized simulated annealing (GSA) method, which is efficient for the parameter estimation. In the method, it is enough to set only the initial parameter distribution and cooling schedule (i.e. characterization of the convergence from the initial parameter distribution in the optimization). Moreover, by setting the initial distribution to take a wide parameter space with an appropriate cooling schedule, we are able to obtain global optima easily and substantially reduce computational costs. Hereafter in this subsection, let us explain our method in detail.

Let a $D$-dimensional vector $\theta$ stand for unknown parameters and $l(\theta)$ the log-likelihood function defined in Appendix 1 for a given model.

In order to maximize this log-likelihood function with respect to parameters, simulated annealing is an appropriate method rather than other (deterministic) numerical optimizations, because there exist substantial Monte Carlo noises in the likelihood computed by the Monte Carlo filter.

In particular, we utilize the following generalized simulated annealing (GSA) method proposed by Tsallis and Stariolo (1996) for estimation of the parameters in the maximal likelihood. Let us define $g_{q_{V}}\left(\Delta \theta_{s}\right)$ as a proposal distribution of $\Delta \theta_{s}$ in a transition, $\theta_{s+1}:=\theta_{s}+\Delta \theta_{s}$. Then, adopting the equation (21) in Tsallis and Stariolo (1996), we suppose

$$
g_{q_{V}}\left(\Delta \theta_{s}\right)=t_{\nu}\left(0_{D}, \frac{\left[T_{q_{V}}^{V}(s)\right]^{2 /\left(3-q_{V}\right)}}{\nu\left(q_{V}-1\right)} I_{D}\right), \nu=\frac{2}{q_{V}-1}-1
$$


with

$$
T_{q_{V}}^{V}(s)=T_{q_{V}}^{V}(1) \frac{2^{q_{V}-1}-1}{(1+s)^{q_{V}-1}-1},
$$

where $t_{\nu}(\mu, \Sigma)$ denotes a (central) $D$-dimensional $t$-distribution with location $\mu$, scale matrix $\Sigma$ and freedom $\nu$. $\left(0_{D}\right.$ and $I_{D}$ denote the $D$-dimensional zero vector and the $D \times D$ identity matrix, respectively.) $T_{q_{V}}^{V}(1)$ is a given constant.

Also, when $l\left(\theta_{s}\right)>l\left(\theta_{s+1}\right)$, following the equation (5) with some remarks in p.398 of Tsallis and Stariolo (1996), we set the acceptance rate of the sample $\theta_{s+1}$ drawn from the proposal distribution as follows:

$$
\begin{gathered}
P_{q_{A}}\left(\theta_{s} \rightarrow \theta_{s+1}\right)=\left[1+\left(q_{A}-1\right) \frac{l\left(\theta_{s}\right)-l\left(\theta_{s+1}\right)}{T_{q_{A}}^{A}(s)}\right]_{+}^{-1 /\left(q_{A}-1\right)}, \\
T_{q_{A}}^{A}(s)=T_{q_{V}}^{V}(s)
\end{gathered}
$$

where $[x]_{+}:=\max \{x, 0\}$.

For our empirical analysis in the next section, we select $q_{A}=1.1, q_{V}=$ 2.5 and $T_{q_{V}}^{V}(1)=1.5$ by searching the optimal values from the following numerical tests.

Firstly, in order to determine a constant $T_{q_{V}}^{V}(1)$ that is able to provide an appropriate search interval, let us consider the $99 \%$ confidence interval of $g_{1}\left(\Delta \theta_{1}\right)$, an initial proposed distribution for the case of $q_{V}=1$ (normal distribution). Because the tail of probability density function is fatter when $q_{V}>1$, considering the case of $q_{V}=1$ is sufficient to obtain an adequate search interval. Specifically, since the differences between initial and optimized parameters rarely become larger than 2 and the $99 \%$ quantile of $g_{1}\left(\Delta \theta_{1}\right)$ is 2.01 for $T_{1}^{V}(1)=1.5$ (i.e. $T_{q_{V}}^{V}(1)$ with $q_{V}=1$ is 1.5 ), we set $T_{q_{V}}^{V}(1)=1.5$ for all $q_{V}$.

Furthermore, referring to the result for the numerical experiments in Tsallis and Stariolo (1996), we implement the optimization with four combinations of the values for the pair $\left(q_{A}, q_{V}\right)$ drawn from $q_{A}=\{1.1,-5.0\}$ and $q_{V}=\{2.5,2.7\}$, in order to find which parameter set is able to provide 
an efficient maximization of the log-likelihood function in each (optimization) step. As a result, we confirm that $\left(q_{A}, q_{V}\right)=(1.1,2.5)$ gives the most efficient optimization, regardless of the input data.

\section{Empirical Analysis}

This section explains the results of an empirical analysis using data of three Japanese mutual funds ${ }^{2}$ and style indexes. We select data of three equity funds and six standard style indexes as follows: Three funds are A: Nissei TOPIX index fund ${ }^{3}$ (Feb. 2003 - Sep. 2015), B: Nomura Japanese equity strategic fund ${ }^{4}$ (Sep. 2000 - Sep. 2015), C: Active Nippon ${ }^{5}$ (Nov. 1999 - Sep. 2015), and six standard style indexes consist of Russell-Nomura's SV(small-value), SG(small-growth), MV(mid-value), MG(mid-growth), TV(topvalue) and TG(top-growth) ${ }^{6}$. Figures 1,2 and Table 1 show these data and the correlation matrix respectively. We notice that there exist high correlations among the style indexes.

\footnotetext{
${ }^{2}$ These data are downloaded from web pages of "Morningstar".

${ }^{3}$ Nissei Asset Management

${ }^{4}$ Nomura Asset Management

${ }^{5}$ Daiwa Asset Management

${ }^{6}$ These indexes are developed by Financial Engineering \& Technology Research Center of Nomura Securities and Russel Investment. The intellectual property right and any other rights, in Russell/Nomura Japan Equity Index belong to Nomura Securities Co., Ltd. ("Nomura") and Frank Russell Company ("Russell"). Nomura and Russell do not guarantee accuracy, completeness, reliability, usefulness, marketability, merchantability or fitness of the Index, and do not account for business activities or services that any index user and/or its affiliates undertakes with the use of the Index.
} 


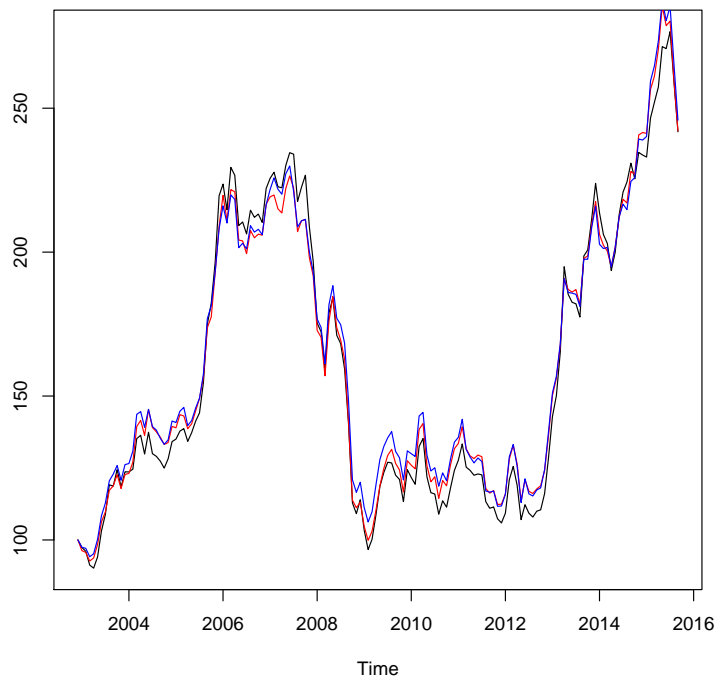

Figure 1: Fund price (blue: A, red: B, black: C, Dec.2002=100) 


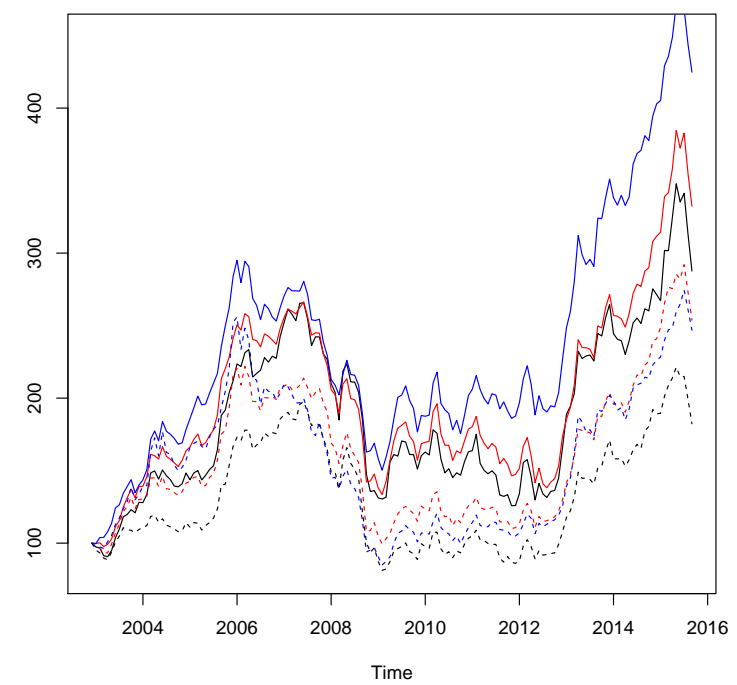

Figure 2: Standard Style Indices (blue: SMALL, red: MID, black: TOP, solid: VALUE, dots: GROWTH, Dec.2002=100) 


\begin{tabular}{c|ccccccccc}
\hline & Fund A & Fund B & Fund C & TV & TG & MV & MG & SV & SG \\
\hline Fund A & 1.00 & 0.99 & 0.97 & 0.95 & 0.96 & 0.97 & 0.96 & 0.93 & 0.88 \\
Fund B & 0.99 & 1.00 & 0.97 & 0.93 & 0.96 & 0.96 & 0.97 & 0.93 & 0.90 \\
Fund C & 0.97 & 0.97 & 1.00 & 0.90 & 0.94 & 0.94 & 0.96 & 0.92 & 0.90 \\
TV & 0.95 & 0.93 & 0.90 & 1.00 & 0.90 & 0.92 & 0.86 & 0.84 & 0.76 \\
TG & 0.96 & 0.96 & 0.94 & 0.90 & 1.00 & 0.90 & 0.93 & 0.85 & 0.81 \\
MV & 0.97 & 0.96 & 0.94 & 0.92 & 0.90 & 1.00 & 0.93 & 0.94 & 0.86 \\
MG & 0.96 & 0.97 & 0.96 & 0.86 & 0.93 & 0.93 & 1.00 & 0.91 & 0.92 \\
SV & 0.93 & 0.93 & 0.92 & 0.84 & 0.85 & 0.94 & 0.91 & 1.00 & 0.92 \\
SG & 0.88 & 0.90 & 0.90 & 0.76 & 0.81 & 0.86 & 0.92 & 0.92 & 1.00 \\
\hline
\end{tabular}

Table 1: Correlation among funds' returns and returns of standard style indexes

\subsection{Analysis with Standard Style Indexes}

A significant feature in this analysis is that since we obtain annual (or semiannual) investment reports of the target mutual funds ${ }^{7}$, we know annually or semi-annually, the exact compositions of stocks in the funds and which style indexes each stock belongs to. Thus, we are able to calculate the true weights, and check periodically (annually or semi-annually) how close to the true ones our estimated style weights are. Here, we remark that each time- $t$ true weight for a style represents the ratio of time- $t$ total values of stocks classified into the corresponding style relative to the time- $t$ fund value.

Moreover, if each portfolio of the stocks classified into each style replicates the corresponding style very well, then the fund can be regarded as a portfolio of the standard style indexes, that is the observation equation (1) is valid, and we are able to evaluate our method based on the equation. Hereafter, we first implement the analysis under the assumption that the equation (1) holds and come back to the issue whether the assumption is

\footnotetext{
${ }^{7}$ We have obtained the data in the reports relevant to our analysis from Nomura Research Institute.
} 
appropriate.

Next, let us explain our model for this analysis, which is a model with jump system noise. Firstly, regarding the system model in this analysis, we combine two models introduced as the examples (i) and (ii) in the previous section. In particular, we assume that

$$
\begin{gathered}
Y_{i t}^{\prime}=f_{i}\left(Y_{t-\Delta t}, \beta_{t-\Delta t}, t\right)+v_{i t}=\log \left(\beta_{i, t-\Delta t}\right)+v_{i t}, \\
Y_{i t}=\frac{e^{Y_{i t}^{\prime}}}{\sum_{i=1}^{n} e^{Y_{i t}^{\prime}}}+w_{i t}, i=1, \cdots, n
\end{gathered}
$$

where each system noise $v_{i t}, w_{i t}$ follows

$$
\left\{\begin{array}{l}
v_{i t} \sim N\left(0, \sigma_{i}^{2}\right) \\
w_{i t}=\sum_{j=1}^{N} x_{j}, N \sim \operatorname{Pois}(\lambda), x_{j} \sim N\left(0, c^{2}\right) .
\end{array}\right.
$$

Here, $\lambda$ is fixed as 0.06 in order to avoid an identification problem, and we confirm that the selection of $\lambda$ does not affect the results of estimating the weights.

This system model describes that each $Y_{i}^{\prime}$ is generated around $\beta_{i}$ in the previous period by adding a system noise. Then, we normalize $Y_{t}^{\prime}$ and add a jump noise. Moreover, we generate

$$
\beta_{t}^{(j)} \equiv\left(\beta_{1 t}, \cdots, \beta_{j-1 t}, \beta_{j+1 t}, \cdots, \beta_{n t}\right),
$$

which follows a conditional distribution expressed as the equations (8) and (15) by using rejection method to select samples satisfying the condition (7).

Here, the rejection method is a statistical method: it takes the sample which satisfies the conditions, while discarding one that does not satisfy those conditions until a sufficient number of samples are obtained.

We also assume that the observation noise follows a i.i.d.(independent identically distributed) normal distribution in the equation (9), and estimate state variables by Monte Carlo filtering. We utilize the generalized simulated annealing (GSA) method introduced in the previous section for estimating parameters by maximization of a likelihood of this model. 
One of the reasons to take this model is that it is a natural extension of a random walk, which is neutral in model selection in a sense that it has no bias for the changes in the weights from the current period to the next period as long as the constraints are satisfied. That is, the weights in the next period is modeled around those in the current period with a noise including jumps, which is expected to capture sudden changes in the weights.

Firstly, we report the estimated parameters in Table 2. We notice that there are some difference in estimated standard deviations of system noises between our model (which we call the jump model, hereafter) and the nonjump model $\left(w_{i t} \equiv 0\right.$ in (15)). Because the estimated standard deviations of the jump term, $c$ are relatively large, the jump model seems to be effective for all three funds.

(a) Jump Model

\begin{tabular}{c|ccccccc}
\hline & TV & TG & MV & MG & SV & SG & Jump \\
Fund & $\sigma_{1}$ & $\sigma_{2}$ & $\sigma_{3}$ & $\sigma_{4}$ & $\sigma_{5}$ & $\sigma_{6}$ & $c$ \\
\hline A & 0.107 & 0.020 & 0.056 & 0.109 & 0.043 & 0.314 & 0.936 \\
B & 0.064 & 0.020 & 0.191 & 0.075 & 0.234 & 0.027 & 0.996 \\
C & 0.217 & 0.512 & 0.055 & 0.050 & 1.341 & 0.111 & 0.776 \\
\hline
\end{tabular}

(b) Non-jump Model $\left(w_{i t} \equiv 0\right.$ in (15))

\begin{tabular}{c|ccccccc}
\hline & TV & TG & MV & MG & SV & SG & Jump \\
Fund & $\sigma_{1}$ & $\sigma_{2}$ & $\sigma_{3}$ & $\sigma_{4}$ & $\sigma_{5}$ & $\sigma_{6}$ & $c$ \\
\hline A & 0.028 & 0.002 & 0.137 & 0.192 & 0.010 & 0.083 & - \\
B & 0.068 & 0.052 & 0.079 & 0.130 & 0.282 & 0.425 & - \\
C & 0.322 & 0.230 & 0.110 & 0.154 & 1.387 & 0.131 & - \\
\hline
\end{tabular}

Table 2: Estimated standard deviation of system noises 
We also show the convergence of our generalized simulated annealing (GSA) for the case of Fund B in Figure 3 and 4 . Those figures show that the convergence is completed and the speed is very fast, which demonstrates the validity of our new estimation method. We obtain similar results for Funds $\mathrm{A}$ and $\mathrm{C}$ and hence, omit to show the results.

$$
(-1 \times) \text { Log-likelihood }
$$
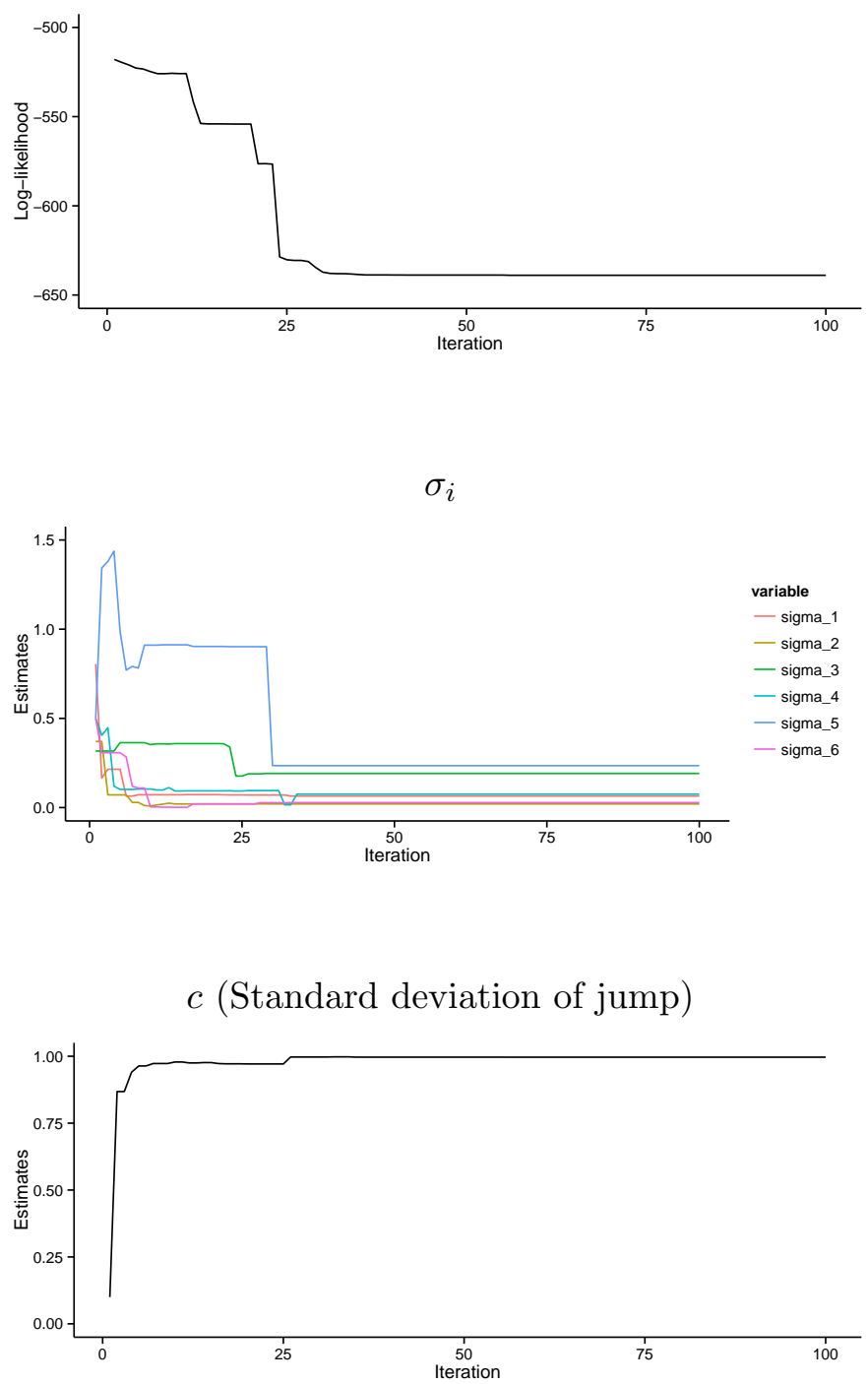

Figure 3: Convergence of Generalized Simulated Annealing for Fund B (1100) 
$(-1 \times)$ Log-likelihood

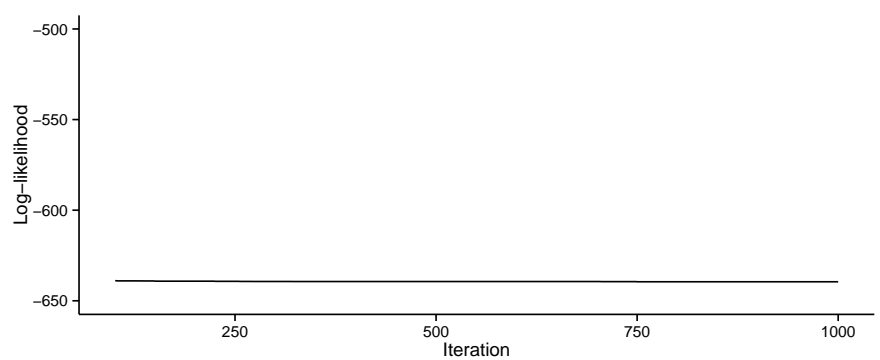

$\sigma_{i}$

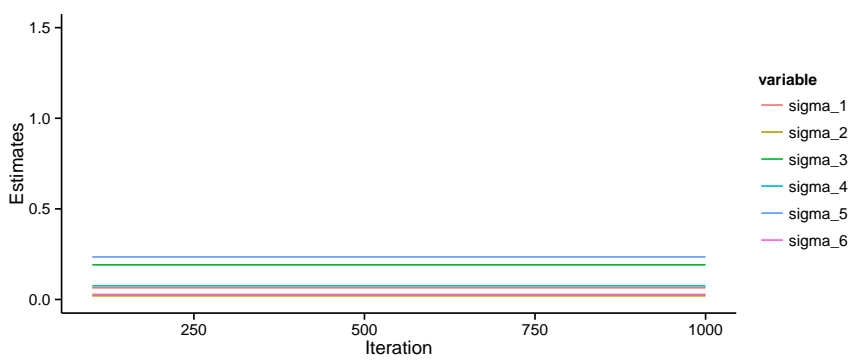

$c$ (Standard deviation of jump)

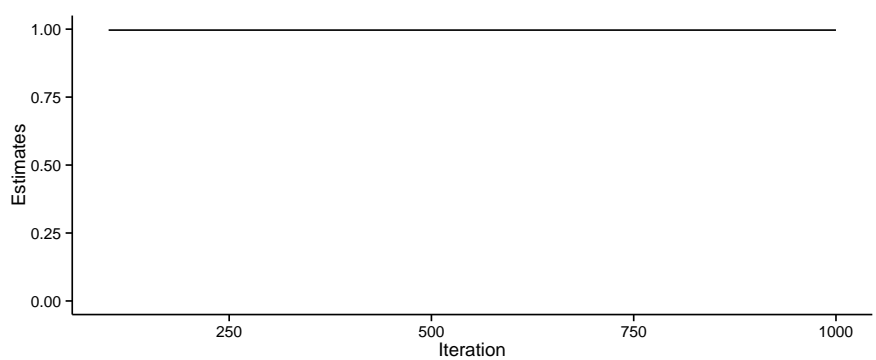

Figure 4: Convergence of Generalized Simulated Annealing for Fund B (1011000)

Next, Figure 5-7 show the results for the smoothing estimated weights of the indexes with the true weights for each fund. In the results of our state space models, we use a fixed-lag smoother ${ }^{8}$ proposed by Kitagawa and Sato

\footnotetext{
${ }^{8}$ We set the lag to be 10 .
} 
(2001). For comparative purpose, we also implement a non-jump model (i.e. $w_{i t} \equiv 0$ in (15)) as well as a window regression. ${ }^{9}$

Comparing the estimated weights with the true ones in each graph, we can observe that the estimates by the Monte Carlo filter are better than those by the window regression.

(a) True weights

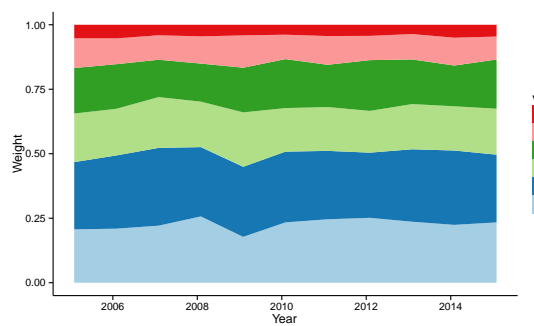

(c) Window regression

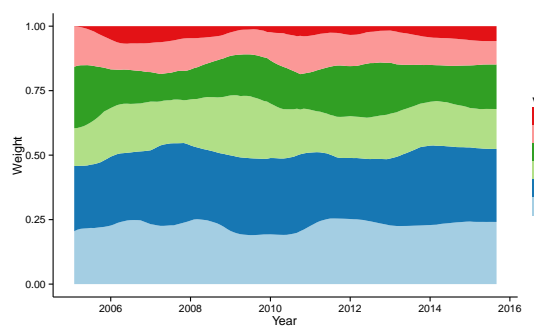

(b) MC filter with Jump Model

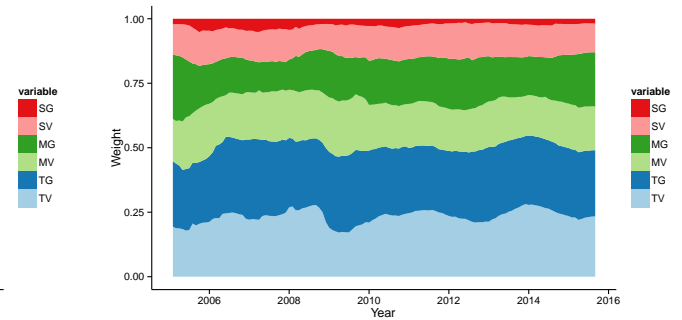

(d) MC filter with Non-Jump model

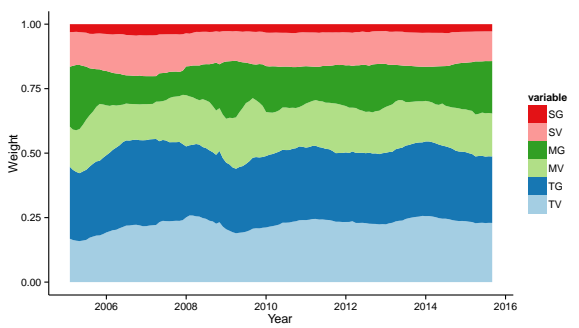

(Model with $w_{i t} \equiv 0$ in (15))

Figure 5: Smoothing results for Fund A

\footnotetext{
${ }^{9}$ We use window-regression with past 24 months. Results are shown only from the second year because the estimated weights by a window regression are not obtained for the initial period.
} 
(a) True weights

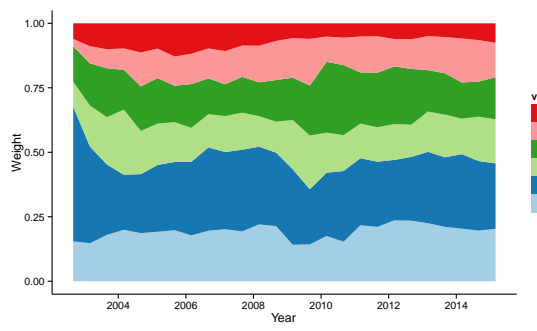

(c) Window regression

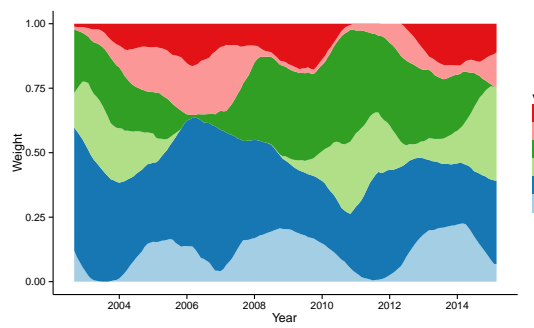

(b) MC filter with Jump Model

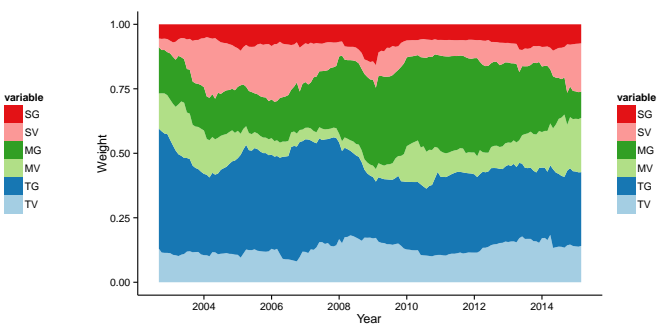

(d) MC filter with Non-Jump model

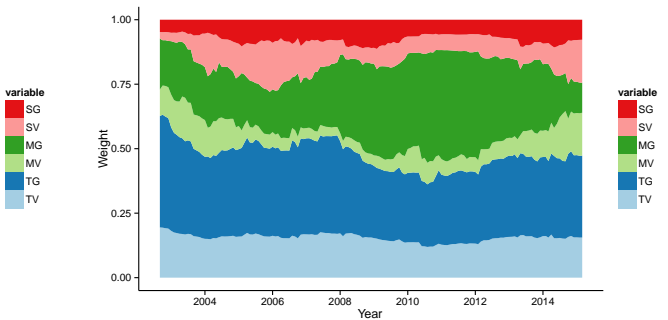

(Model with $w_{i t} \equiv 0$ in (15))

Figure 6: Smoothing results for Fund B 
(a) True weights

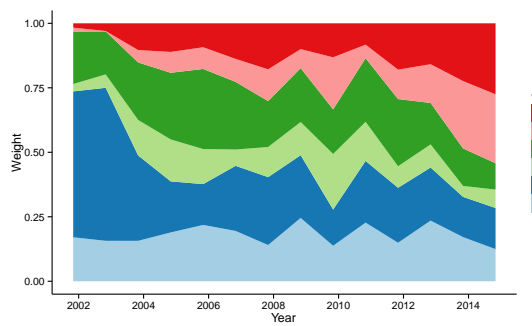

(c) Window regression

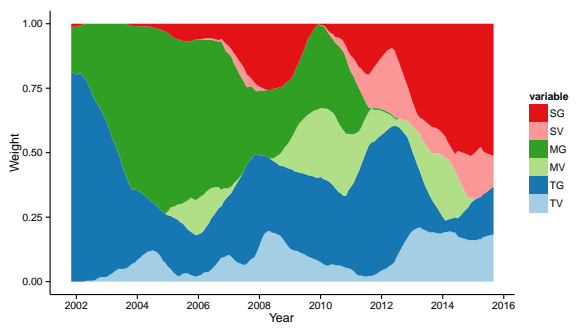

(b) MC filter with Jump Model

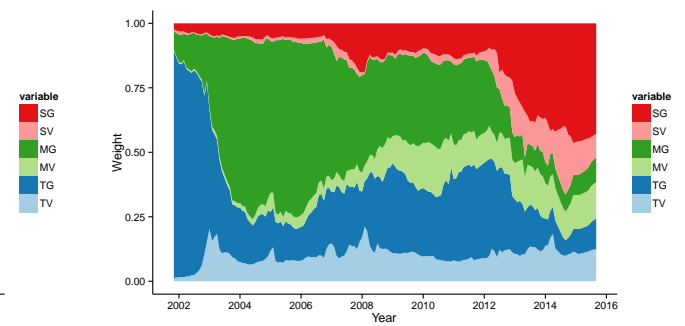

(d) MC filter with Non-Jump model

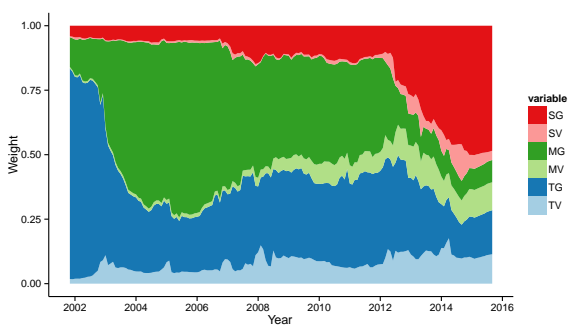

(Model with $w_{i t} \equiv 0$ in (15))

Figure 7: Smoothing results for Fund C

We also calculate Mean Absolute Error (MAE) defined below for a quantitative comparison of the estimated weights $\beta_{i t}$.

$$
M A E:=\frac{1}{N_{\mathcal{T}_{0}}} \sum_{i=1}^{6} \sum_{t \in \mathcal{T}_{0}}\left|\beta_{i t}^{\text {estimate }}-\beta_{i t}^{\text {true }}\right|,
$$

where $\mathcal{T}_{0}$ is the set of observed periods of the true weights and $N_{\mathcal{T}_{0}}$ is the total number of $t$ in the set. MAE shows the absolute difference between the true weights and the estimated weights per a time period, and it is expected that MAE becomes smaller for the better estimation. Tables 3-5 show the results, which indicate our method with jump model works better than the window regression. 


\begin{tabular}{c|cccccc|c}
\hline & TV & TG & MV & MG & SV & SG & MAE \\
\hline MC Filter for jump model & 0.014 & 0.010 & 0.009 & 0.017 & 0.022 & 0.017 & 0.090 \\
MC Filter for non-jump model & 0.014 & 0.012 & 0.017 & 0.029 & 0.030 & 0.012 & 0.115 \\
Window regression & 0.013 & 0.016 & 0.015 & 0.024 & 0.021 & 0.015 & 0.105 \\
\hline
\end{tabular}

Table 3: Mean absolute error of estimated weights for Fund A

TV: top value, TG: top growth, MV: mid value, MG: mid growth, SV: small value, SG: small growth.

Non-Jump model: the model without jump-noise in (15).

\begin{tabular}{c|cccccc|c}
\hline & TV & TG & MV & MG & SV & SG & MAE \\
\hline MC Filter for jump model & 0.063 & 0.058 & 0.059 & 0.079 & 0.059 & 0.023 & 0.343 \\
MC Filter for non-jump model & 0.043 & 0.050 & 0.071 & 0.097 & 0.049 & 0.023 & 0.334 \\
Window regression & 0.084 & 0.088 & 0.094 & 0.098 & 0.074 & 0.049 & 0.487 \\
\hline
\end{tabular}

Table 4: Mean absolute error of estimated weights for Fund B

\begin{tabular}{c|cccccc|c}
\hline & TV & TG & MV & MG & SV & SG & MAE \\
\hline MC Filter for jump model & 0.082 & 0.080 & 0.057 & 0.163 & 0.068 & 0.053 & 0.504 \\
MC Filter for non-jump model & 0.103 & 0.090 & 0.075 & 0.178 & 0.094 & 0.063 & 0.603 \\
Window regression & 0.100 & 0.098 & 0.068 & 0.187 & 0.073 & 0.081 & 0.608 \\
\hline
\end{tabular}

Table 5: Mean absolute error of estimated weights for Fund C 
Next, we report the AICs for our jump model and non-jump model in Table 6. The jump model gives better AIC for Fund C, while the non-jump model does better for Fund A and B. Particularly, for Fund A, MAEs are small and similar by these three methods, because this fund is an index fund and its weights do not change largely. On the other hand, since Fund B and $\mathrm{C}$ are active fund and their weights change largely, our methods become effective.

\begin{tabular}{c|ccc}
\hline & Fund A & Fund B & Fund C \\
\hline Jump model & -1466.81 & -1260.952 & -1049.056 \\
Non-jump model & -1472.604 & -1264.408 & -1048.512 \\
\hline
\end{tabular}

Table 6: AIC for jump model and non-jump model (Model without jumpnoise in (15))

However, there is still significant difference between the true weights and estimated weights in Fund C. Then, in order to examine the reason we simulate the returns by using the true weights with standard style indexes and compare them with actual returns of Funds A, B and C. The correlations between them for Funds $\mathrm{A}$ and $\mathrm{B}$ are $0.999\left(R^{2}\right.$ is 0.998.) and $0.992\left(R^{2}\right.$ is 0.984.), respectively. Figure 8 shows Fund A, B and C prices relative to the initial price of each observation respectively. Because fund's actual and simulated returns are highly correlated for Fund A and Fund B, simulated prices are close to actual prices. On the other hand, the correlation for Fund $\mathrm{C}$ is $0.978\left(R^{2}\right.$ is 0.957 .) although it is expected to be close to one. Then, in Figure 8 , we observe more differences between actual prices and simulated prices in Fund C than those in Fund A and B. 
(a) Fund A

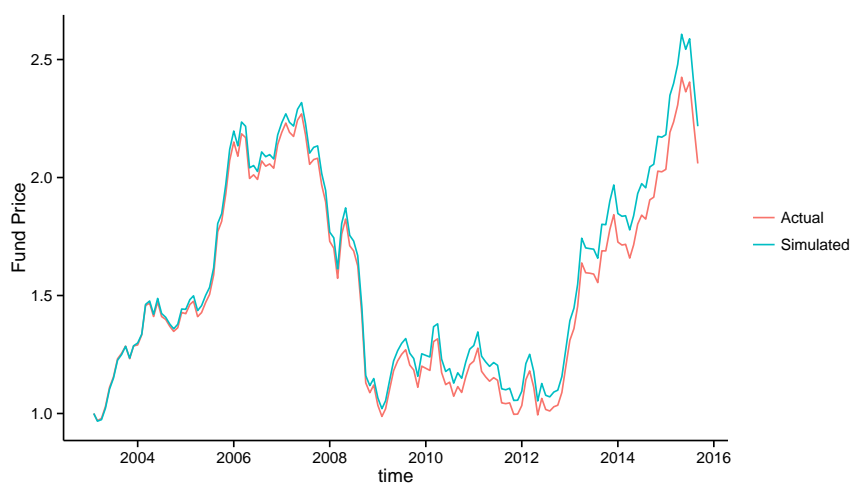

(b) Fund B

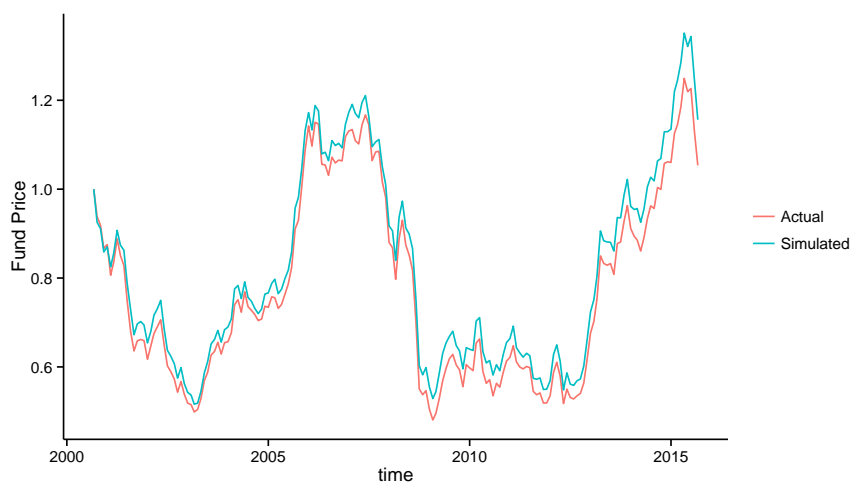

(c) Fund C

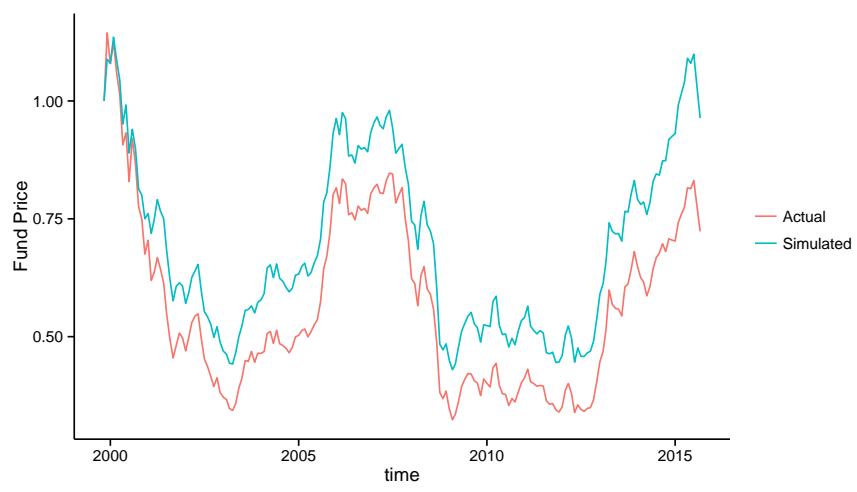

Figure 8: Fund prices simulated with actual and simulated returns

In addition, we have observed from Table 3-5 that the MAEs for Funds A 
and $\mathrm{B}$ which exhibit high correlations are much smaller than the MAEs for Fund C. Judging from these results, we may conclude that the assumption that the fund can be approximated by a portfolio of the standard six style indexes is appropriate for Funds A and B, while it is less appropriate for Fund C. The next subsection will investigate this point further.

\subsection{Analysis with New Style Indexes}

In order to improve style estimates especially for Fund C, we create fundspecific style index returns, denoted by $I_{i, t}^{\text {(fund) }}$ of style $i \in\{\mathrm{TV}, \mathrm{TG}, \mathrm{MV}, \mathrm{MG}, \mathrm{SV}, \mathrm{SG}\}$ at observation time $t$. Moreover, let us define $\mathcal{F}_{a(t)}^{(\text {fund })}$ as the set of stocks invested by "(fund)" at $a(t)$, where each stock is labeled by different $j \in \mathbf{N}$ (the set of natural numbers) in the set $\mathcal{F}_{a(t)}^{(\text {fund) }}$, and $a(t)$ is the latest time point when the fund's report is released before time $t$, that is

$$
a(t)=\max _{k}\left\{a_{k}\right\} \text { such that } a_{k}<t .
$$

Here, $\left\{a_{k}\right\}$ denotes a set of time points when the fund's investment reports are released annually or semi-annually. We also define $\mathcal{G}_{b(t)}^{(i)}$ as the set of stocks classified into style $i$ at $b(t)$, where $b(t)$ is the latest time point when the style classification table $\mathrm{e}^{10}$ is released before time $t$, that is

$$
b(t)=\max _{k}\left\{b_{k}\right\} \text { such that } b_{k}<t
$$

$\left\{b_{k}\right\}$ denotes a set of time points when the style classification reports are released annually. Then, we can express the subset of stocks held by "(fund)" and classified into style $i$ as follows:

$$
\mathcal{J}_{t}^{(\text {fund }, i)}=\left\{j \mid j \in \mathcal{F}_{a(t)}^{(\text {fund })} \cap \mathcal{G}_{b(t)}^{(i)}\right\} .
$$

Now, our new index return $I_{i, t}^{\text {(fund) }}$ of style $i$ at time $t$ for "(fund)" is defined as an weighted average of these stocks' returns, where the stock $j$ 's weight are assumed to be determined by the $j$ 's weights in the fund at time

\footnotetext{
${ }^{10}$ This table is released by Financial Engineering \& Technology Research Center of Nomura Securities in every November.
} 
$a(t)$, denoted as $w_{j, a(t)}^{(\text {fund })}\left(j \in \mathcal{F}_{a(t)}^{(\text {fund })}, \sum_{j} w_{j, a(t)}^{(\text {fund })}=1\right)$. We note that those weights are also obtained in the fund's investment reports.

Thus, we define a new fund-specific style index $i$ 's return $I_{i, t}^{\text {(fund) }}$ as

$$
I_{i, t}^{(\text {fund })}=\frac{\sum_{j \in \mathcal{J}_{t}^{(\text {fund }, i)}} w_{j, a(t)}^{(\text {fund })} r_{j, t}}{\sum_{j \in \mathcal{J}_{t}^{(\text {fund }, i)}} w_{j, a(t)}^{(\text {fund })}},
$$

where $r_{j, t}$ denotes stock $j$ 's total return at time $t$.

Next, we calculate such new index returns for Funds B and C, which are denoted by $I_{i, t}^{\text {(FundB) }}$ and $I_{i, t}^{(\text {FundC })}, i \in\{\mathrm{TV}, \mathrm{TG}, \mathrm{MV}, \mathrm{MG}, \mathrm{SV}, \mathrm{SG}\}$, respectively. Then, the absolute differences between actual and simulated returns are shown in Figure 9. These figures show that the differences are smaller than those simulated from the standard six style indexes used in the previous subsection. Also, the correlations between actual and simulated returns become higher as $0.996\left(R^{2}\right.$ is 0.993$)$ for Fund $\mathrm{B}$ and $0.981\left(R^{2}\right.$ is 0.963$)$ for Fund C. 
(a) Fund B

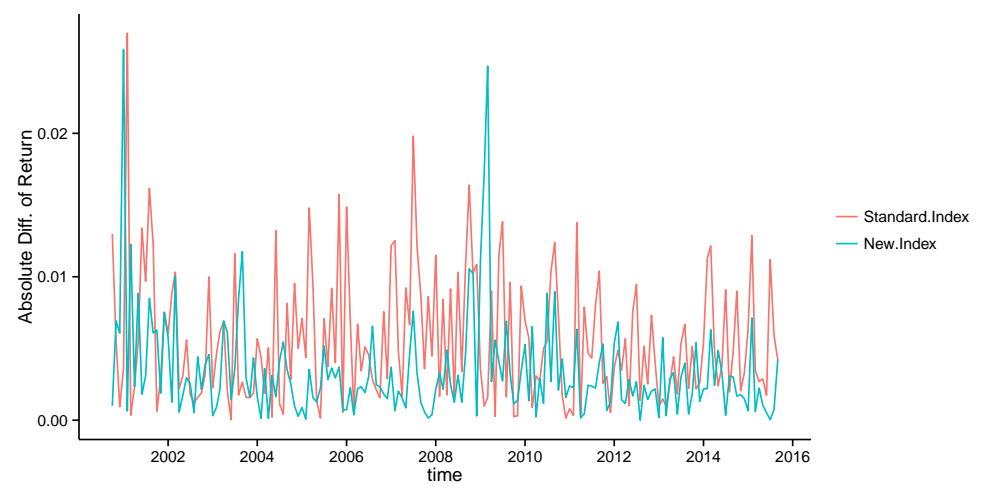

(b) Fund C

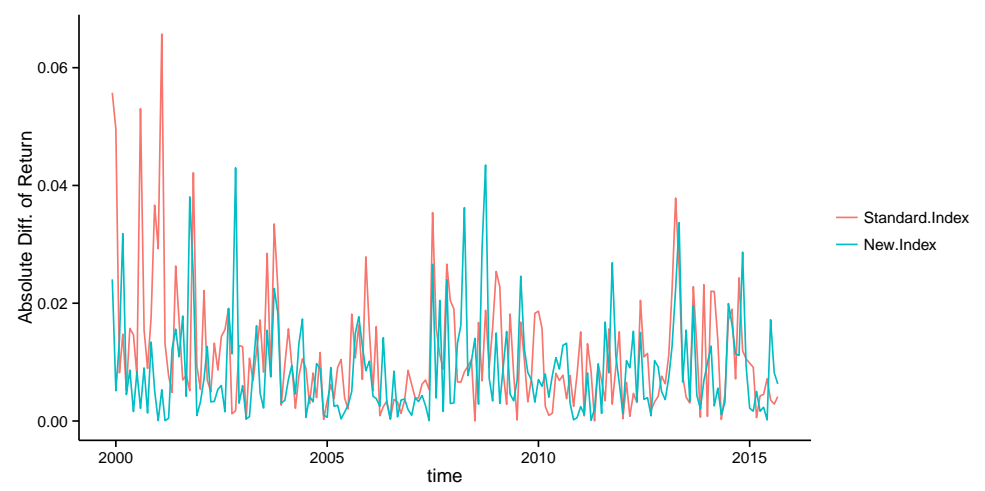

Figure 9: Absolute difference between actual and simulated returns

Moreover, we present the funds' prices calculated from actual and simulated returns in Figure 10. This figure shows that the simulated prices with the new index returns $I_{i, t}^{(\text {FundB) }}$ are a bit higher than the actual ones for Fund B, mainly due to changes in the weights between two consecutive timings when the fund's investment reports are released, or/and the fund management fees and transaction costs paid from the fund's asset. Figure 10 also demonstrates that simulated Fund $\mathrm{C}$ prices with the new index returns $I_{i, t}^{(\text {FundC) }}$ get closer to actual prices, which implies that our new indexes successfully explain the Fund C's returns. 
(a) Fund B

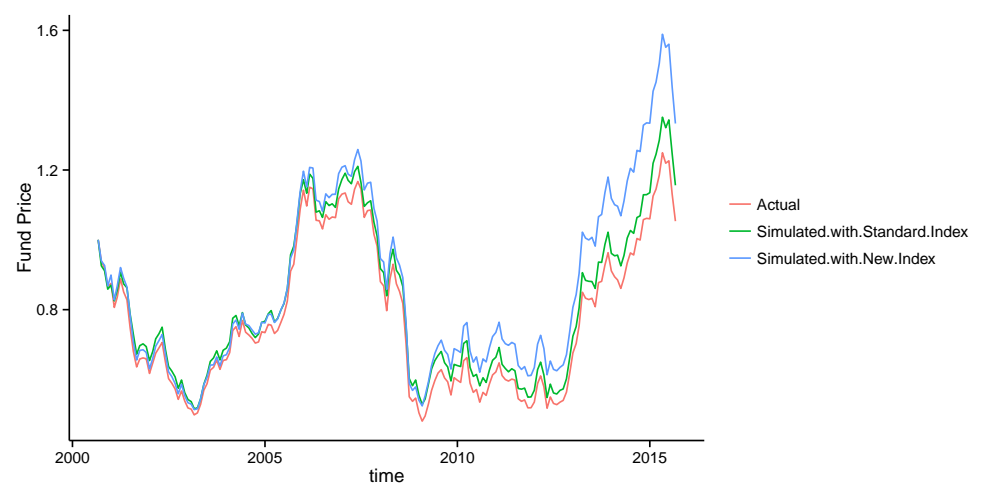

(b) Fund C

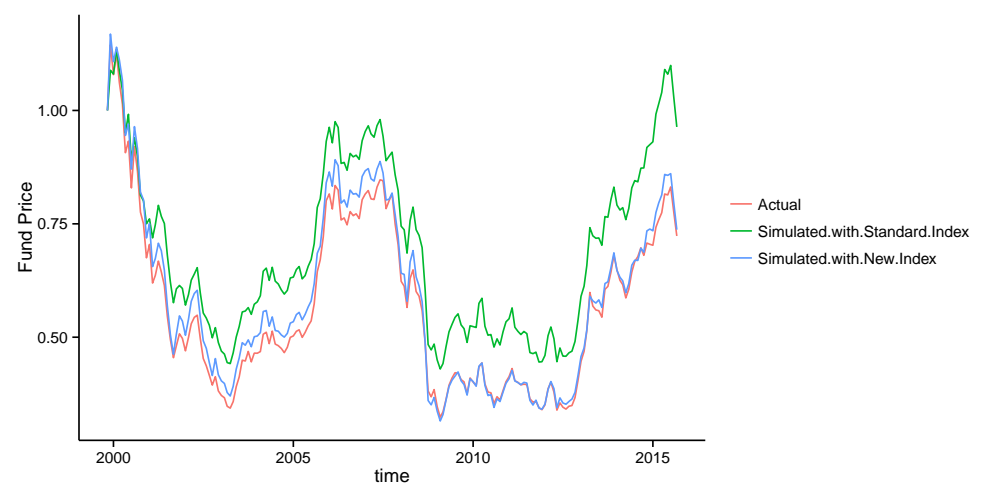

Figure 10: Fund prices simulated with actual and simulated returns using standard and new index

Next, we re-estimate style weights and re-calculate MAEs and AICs using these indexes as explanation variables. The estimates for the style weights are shown in Figure 11 and 12 for Fund B and Fund C, respectively. Table 7 and 8 show MAEs for Funds B and C, respectively.

It is observed in Figure 11 and 12 that we can identify the weights among growth and value styles of small and middle caps (i.e. SG,SV,MG,MV in the figures), much better than in the previous section using the standard style indexes. In particular, over-estimating results for the weight of middle growth are resolved for both of Fund B and Fund C, because the new indexes 
do not reflect price changes of the stocks that are not held by the fund. As a result, the correlation between middle value index and middle growth index becomes smaller. For example, the correlation between $I_{\mathrm{MV}, t}^{(\mathrm{FundC})}$ and $I_{\mathrm{MG}, t}^{(\text {Fund })}$ is 0.686 which is lower than that for the standard indexes, $0.819^{11}$. This implies that we are able to avoid bias caused by multi co-linearity among explanation variables.

In Table 7 and 8, we can observe that MAEs become smaller than the ones when we use the standard style indexes in the previous subsection. Moreover, we emphasize that the MC filter method provides the better results than the window regression, particularly, MC filter with jumps gives the best. Also, AICs are presented in Table 9, where the better results are obtained by applying our new indexes.

In sum, we have found that existing standard six indexes are not necessarily appropriate in the style analysis for some mutual funds, especially for Fund $\mathrm{C}$ in our example. Even for such funds, we are able to create more adequate ones based on the funds' investment reports, and confirm that our method with jump system noises is very effective in estimation of the style weights based on the new indexes.

${ }^{11}$ These results are estimated using the observations for Fund C from Nov. 1999 to Sep. 2015 
(a) True weights

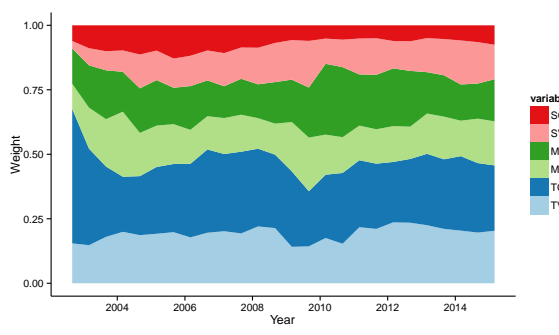

(c) Window regression

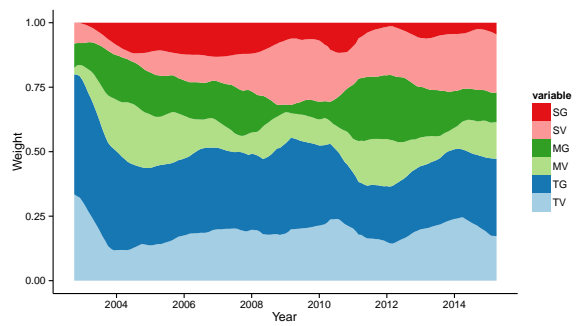

(b) MC filter with Jump Model

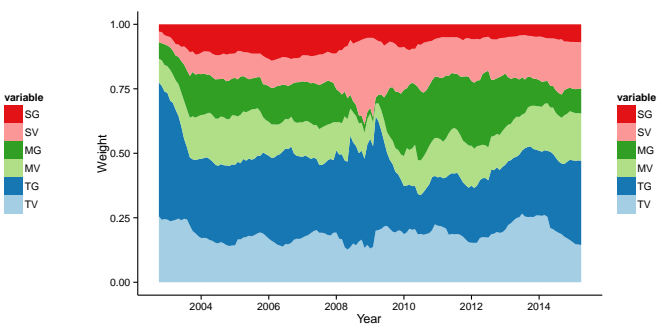

(d) MC filter with Non-Jump model

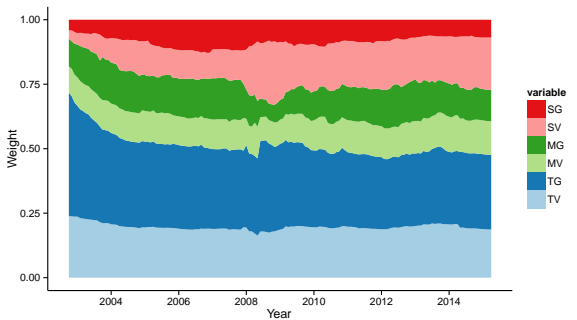

(Model with $w_{i t} \equiv 0$ in (15))

Figure 11: Smoothing results for Fund B using new indexes 
(a) True weights

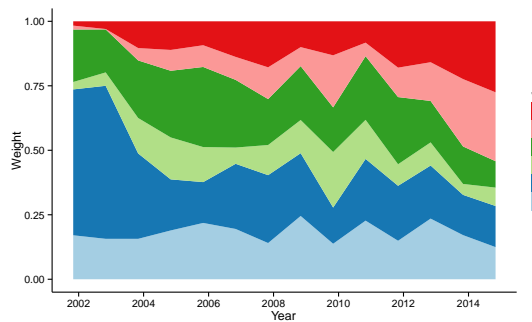

(c) Window regression

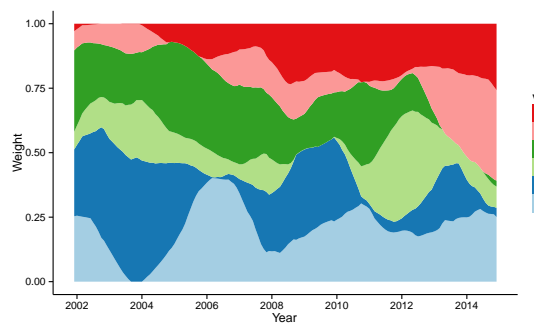

(b) MC filter with Jump Model

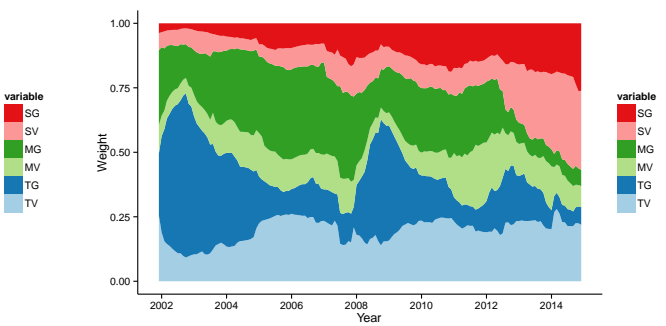

(d) MC filter with Non-Jump model

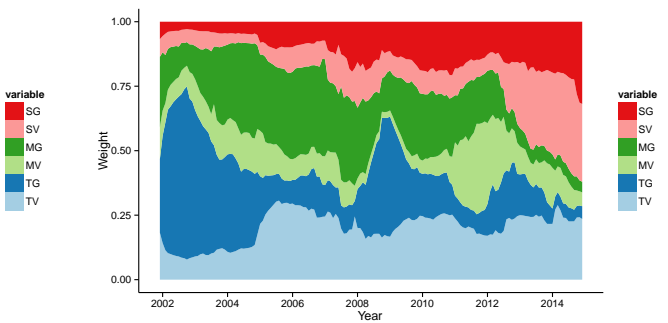

(Model with $w_{i t} \equiv 0$ in (15))

Figure 12: Smoothing results for Fund C using new indexes

\begin{tabular}{c|cccccc|c}
\hline & TV & TG & MV & MG & SV & SG & MAE \\
\hline MC Filter for jump model & 0.040 & 0.043 & 0.026 & 0.040 & 0.028 & 0.012 & 0.189 \\
MC Filter for non-jump model & 0.026 & 0.041 & 0.036 & 0.046 & 0.031 & 0.022 & 0.203 \\
Window regression & 0.046 & 0.044 & 0.040 & 0.045 & 0.051 & 0.024 & 0.251 \\
\hline
\end{tabular}

Table 7: Mean absolute error of estimated weights for Fund B using new indexes 


\begin{tabular}{c|cccccc|c}
\hline & TV & TG & MV & MG & SV & SG & MAE \\
\hline MC Filter for jump model & 0.049 & 0.103 & 0.057 & 0.059 & 0.035 & 0.031 & 0.333 \\
MC Filter for non-jump model & 0.055 & 0.106 & 0.075 & 0.074 & 0.040 & 0.040 & 0.390 \\
Window regression & 0.085 & 0.139 & 0.086 & 0.071 & 0.062 & 0.049 & 0.493 \\
\hline
\end{tabular}

Table 8: Mean absolute error of estimated weights for Fund C using new indexes

\begin{tabular}{c|cc}
\hline & Fund B & Fund C \\
\hline Jump model & -1332.418 & -1120.234 \\
Non-jump model & -1337.774 & -1111.236 \\
\hline
\end{tabular}

Table 9: AIC for jump model and non-jump model : re-estimation on Funds $\mathrm{B}$ and Fund $\mathrm{C}$ using new indexes

Finally, it is noted that despite the similarity of price time series of these three funds shown in Figure 1, our method successfully detects the differences of styles among these funds, which may show a potential of our method to make a new discovery in style analysis of mutual funds.

\section{Conclusion}

We have developed a new method for estimating style weights of mutual funds in a general state space framework with particle filtering, particularly Monte Carlo filter. Moreover, we have successfully applied generalized simulated annealing (GSA) to estimation of the model parameters. To the best of our knowledge, it is a novel estimation method in particle filtering for a statistical application in finance.

We have also implemented empirical analysis by using the data of three Japanese mutual funds and six standard style indexes to confirm advantage of our approach over a window regression, which is frequently applied in 
practice. Further, we have examined annual or semiannual investment reports for the mutual funds in detail, and found that the standard indexes are not necessarily adequate in style analysis, especially for Fund $\mathrm{C}$ in our examples.

Even for such funds, we have created fund-specific style indexes to obtain more accurate estimates of the weights. Then, a numerical experiment has demonstrated that our method with jump system noises works much better than the window regression. Developing new investment strategies based on effective use of the present result will be one of our next research topics.

\section{Appendix 1: Algorithm for Monte Carlo Filter}

This appendix describes the outline of a standard algorithm of Monte Carlo filter based on Kitagawa(1996).

First, we summarize the notations: $p\left(X_{t} \mid Z_{t-\Delta t}\right)$, called "one step ahead prediction" denotes the conditional density function of a state vector $X_{t}$ given an observation vector $Z_{t-\Delta t}$ where $\Delta t$ is the interval of time series data. $p\left(X_{t} \mid Z_{t}\right)$, called "filter" denotes the conditional density function of $X_{t}$ given $Z_{t} \cdot\left\{p_{t}^{[1]}, \cdots, p_{t}^{[m]}\right\}$ and $\left\{\xi_{t}^{[1]}, \cdots, \xi_{t}^{[m]}\right\}$ represent the vectors of the realization of $m$ trials of Monte Carlo simulations from $p\left(X_{t} \mid Z_{t-\Delta t}\right)$ and $p\left(X_{t} \mid Z_{t}\right)$, respectively. Then, if we set $\left\{\xi_{0}^{[1]}, \cdots, \xi_{0}^{[m]}\right\}$ as the realization of Monte Carlo from $p_{0}(X)$, the density function of the initial state vector $X_{0}$, an algorithm of Monte Carlo filter is as follows.

\section{[Summary of Algorithm for Monte Carlo filter]}

1. Generate the initial state vector $\left\{\xi_{0}^{[1]}, \cdots, \xi_{0}^{[m]}\right\}$.

2. Apply the following steps (a) (d) to each time $t=0, \Delta t, 2 \Delta t, \cdots,\left(T_{*}-\right.$ $\Delta t), T_{*}$ where $T_{*}$ denotes the final time point of the data.

- (a) Generate the system noise $v_{t}^{[k]}, k=1, \cdots, m$ according to the 
density function $q(v)$.

- (b) Compute for each $k=1, \cdots, m$

$$
p_{t}^{[k]}=F\left(\xi_{t-\Delta t}^{[k]}, v_{t}^{[k]}\right)(\text { the system model in }(2))
$$

or

$$
p_{t}^{[k]} \sim \mathbf{F} \mid \xi_{t-\Delta t}^{[k]}(\text { the system model in }(3)) .
$$

- (c) Evaluate the density function of $\psi(u)$ at $u=Z_{t}-H\left(p_{t}^{[k]}\right)$, $k=1, \cdots, m$ and define those as $\alpha_{t}^{[k]}, k=1, \cdots, m$.

- (d) Resample $\left\{\xi_{t}^{[1]}, \cdots, \xi_{t}^{[m]}\right\}$ from $\left\{p_{t}^{[1]}, \cdots, p_{t}^{[m]}\right\}$. More precisely, resample each $\xi_{t}^{[k]}, k=1, \cdots, m$ from $\left\{p_{t}^{[1]}, \cdots, p_{t}^{[m]}\right\}$ with the probability given by:

$$
\text { Prob. }\left(\xi_{t}^{[k]}=p_{t}^{[i]} \mid Z_{t}\right)=\frac{\alpha_{t}^{[i]}}{\sum_{k=1}^{m} \alpha_{t}^{[k]}}, k=1, \cdots, m, i=1, \cdots, m .
$$

The estimation of unknown parameters is based on the maximum likelihood method. If $\theta$ denotes the vector representing whole unknown parameters, the likelihood $L(\theta)$ is given by:

$$
\begin{aligned}
& L(\theta)=g\left(Z_{\Delta t}, \cdots, Z_{T_{*}} \mid \theta\right)=\Pi_{i=1}^{\frac{T_{*}}{\Delta t}} g_{i}\left(Z_{i \Delta t} \mid Z_{\Delta t}, \cdots, Z_{(i-1) \Delta t}, \theta\right) \\
& g_{1}\left(Z_{\Delta t} \mid Z_{0}\right)=p_{0}\left(Z_{\Delta t}\right)
\end{aligned}
$$

where $g\left(Z_{\Delta t}, \cdots, Z_{T_{*}} \mid \theta\right)$ and $g_{i}\left(Z_{i \Delta t} \mid Z_{\Delta t}, \cdots, Z_{(i-1) \Delta t}, \theta\right)$ denote the joint density function of $Z_{\Delta t}, \cdots, Z_{T_{*}}$ with parameter vector $\theta$ and the conditional density function of $Z_{i \Delta t}$ given $Z_{\Delta t}, \cdots, Z_{(i-1) \Delta t}$ with $\theta$, respectively. The $\log$-likelihood $l(\theta)$ is computed approximately within the framework of the Monte Carlo filter by:

$$
l(\theta)=\sum_{i=1}^{\frac{T_{*}}{\Delta t}}\left(\log \sum_{k=1}^{m} \alpha_{i \Delta t}^{[k]}\right)-\frac{T_{*}}{\Delta t} \log m .
$$

Then, maximize $l(\theta)$ with respect to $\theta$ to obtain the maximum likelihood estimator $\hat{\theta}$. For optimization, Generalized Simulated Annealing method is applied. Finally, we utilize AIC(Akaike Information Criterion, Akaike 
(1973)) as a criterion to select a model if there are several candidates where AIC is defined by:

$$
\mathrm{AIC}=-2 l(\hat{\theta})+2(\text { the number of parameters }) .
$$

That is, the model with the smaller AIC can be regarded as the better model.

\section{Appendix 2: Detailed Algorithm in Empirical Analysis}

We apply the general algorithm described in Appendix 1 to our empirical analysis as follows:

- First, select $j \in\{1, \cdots, n\}$ with probability $\frac{1}{n}$ for each sample $k=$ $1, \cdots, m$ in 2.(b) of Appendix 1. where $n$ denotes the number of style indexes.

- Next, generate $p_{i t}^{[k]}, k=1, \cdots, m$, the realizations of $Y_{i t}, i \neq j$ from the equation (6). We note that a state vector $X_{t}$ consists of $Y_{t}$ and $\beta_{t}$ in this case.

- Third, generate $p_{i t}^{[k]}, k=1, \cdots, m$, the realizations of $\beta_{i t}, i \neq j$ from the conditional distribution $G\left(y^{(j)}\right)$ given in the equation (8) by using the rejection method to select samples satisfying the condition (7):

$$
A_{t}^{(j)}=\left\{0 \leq Y_{1 t}, \cdots, 0 \leq Y_{j-1 t}, 0 \leq Y_{j+1 t}, \cdots, 0 \leq Y_{n t}, \sum_{i \neq j} Y_{i t} \leq 1\right\} .
$$

Here the rejection method is a statistical method; it takes the sample that satisfies the conditions while discarding one that does not satisfy those conditions until a sufficient number of samples are obtained.

- Finally, $\xi_{i t}^{[k]}, k=1, \cdots, m$ that represent the filters of $Y_{i t}, i \neq j$ and $\beta_{i t}$, $i \neq j$ are determined by the filtering method described in 2.(c) and (d) of Appendix 1. Note that $\xi_{j t}^{[k]}$, the filters of $\beta_{j t}$ is determined by the constraint as:

$$
\beta_{j t}=1-\sum_{i \neq j} \beta_{i t}
$$




\section{References}

[1] Akaike, H. (1973). Information theory and an extension of the maximum likelihood principle, Second International Symposium on Information Theory (eds. B. N. Petrov and F. Csáki), 267-281, Akademiai Kiádo, Budapest.

[2] Annaert, J, and G. Van Campenhout (2007) Time variation in mutual fund style exposures, Review of Finance, 11, 4, 633-661.

[3] Bodson, L., A. Coën, and G. Hübner (2010). DYNAMIC HEDGE FUND STYLE ANALYSIS WITH ERRORS-IN-VARIABLES, Journal of Financial Research, 33, Issue 3, 201-221.

[4] Brown, S. J. and Goetzmann, W. N. (1997). Mutual fund styles, Journal of Financial Economics, 43, 373-399.

[5] Busse, J. A. (1999). Volatility Timing in Mutual Funds: Evidence from Daily Returns, The Review of Financial Studies, 12, 1009-1041.

[6] Chan, L.K.C., Chen, HL. and Lakonishok, J. (2002). On mutual fund investment styles, The Review of Financial Studies, 15, 1407-1437.

[7] Doucet, A., Barat, E. and Duvaut, P. (1995). A Monte Carlo approach to recursive Bayesian state estimation, Proceedings IEEE Signal Processing/Athos Workshop on Higher Order Statistics, Girona, Spain.

[8] Durbin, J. and Koopman, S.J. (1997). Monte Carlo maximum likelihood estimation for non-Gaussian state space models, Biometrika, 84, 669684.

[9] Ferson, W. and Schadt, R. (1996). Measuring fund strategy and performance in changing economic condition, Journal of Finance, 51, 425461. 
[10] Fung, W. and Hsieh, D. A. (1997). Empirical characteristics of dynamic trading strategies: The case of hedge funds, The Review of Financial Studies, 10, 275-305.

[11] Higuchi, T. (1999). Applications of quasi-periodic oscillation models to seasonal small count time series, Computational Statistics $\&$ Data Analysis, 30, Issue 3, 281-301.

[12] Gordon, N., Salmond, D.J. and Smith, A.F.M. (1993). Novel approach to nonlinear/non-Gaussian Bayesian state estimation, IEE Proceedings$F, \mathbf{1 4 0}, 2,107-113$.

[13] Grinblatt, M., Titman, S. and Wermers, R. (1995). Momentum strategies, portfolio performance and herding: A study of mutual fund behavior, American Economic Review, 85, 1088-1105.

[14] Kitagawa, G. (1996). Monte Carlo Filter and Smoother for NonGaussian Nonlinear State Space Models, Journal of Computational and Graphical Statistics, 5, 1, 1-25.

[15] Kitagawa, G. (1998). A Self-Organizing State-Space Model, Journal of the American Statistical Association, 93, 443, 1203-1215.

[16] Kitagawa, G. and Gersch, W. (1996). Smoothness Prior Analysis of Time Series (Lecture Notes in Statistics No. 116), Springer-Verlag, Berlin.

[17] Kitagawa, G. and Sato, S. (2000). Nonlinear state space model approach to financial time series with time-varying variance, Proceedings of the Hong Kong International Workshop on Statistics and Finance: An Interface (eds. W.S. Chan, W.K. Li and H. Tong), Imperial College Press, London.

[18] Kitagawa, G. and Sato, S. (2001). Monte Carlo smoothing and selforganizing state-space model, Sequential Monte Carlo Methods in Prac- 
tice (eds. Doucet, A, de Freitas, N. and Gordon, N.J.), Springer-Verlag, New York.

[19] Kobayashi, T., Sato, S. and Takahashi,A. (2005). Style Analysis Based on a General State Space Model and Monte Carlo Filter, CIRJE Discussion Paper Series, CIRJE-F-337.

[20] Marques, R., A. Pizzinga, and L. Vereda (2012). Restricted Kalman filter applied to dynamic style analysis of actuarial funds, Applied Stochastic Models in Business and Industry, 28, Issue 6, 558-570.

[21] Pattarina,F., Paterlinib, S., Minervac, T. (2004). Clustering financial time series: an application to mutual funds style analysis, Computational Statistics 83 Data Analysis, 47, Issue 2, 353-372.

[22] Sharpe, W. F. (1992). Asset allocation: Management style and performance measurement, The Journal of Portfolio Management, Winter, 7-19.

[23] Swinkels, L. A. P. and van der Sluis, P. J. (2006). Return-Based Style Analysis with Time-Varying Exposures, European Journal of Finance, 12, 529-552.

[24] Takahashi, A. and Sato, S. (2001). Monte Carlo Filtering Approach for Estimating the Term Structure of Interest Rates, Annals of The Institute of Statistical Mathematics, 53, No.1, 50-62.

[25] Tanizaki, H. (1993). Nonlinear Filters (Lecture Notes in Economics and Mathematical Systems, 400), Springer-Verlag, Berlin.

[26] Tsallis, C. and Stariolo, D. A. (1996). Generalized simulated annealing, Physica A: Statistical Mechanics and its Applications, 233, Issues 1-2, 395-406. 